\title{
Decision-making in Arytenoid Granuloma Management: An Insight into the Presentations and Etiology
}

\author{
S Vivek ${ }^{1}$, Jayakumar R Menon ${ }^{2}$, Reshmi M Nair ${ }^{3}$
}

\begin{abstract}
Introduction: Arytenoid granulomas are benign exophytic inflammatory lesions that are found in the posterior glottis. This study aims to address the two presentations of arytenoid granuloma, namely process granuloma (PG) and body granuloma (BG), and to study the etiological factors behind each of these and thereby provide better understanding of the treatment of these two forms.

Materials and methods: Descriptive study was performed by analyzing the videolaryngoscopic (VLS) recordings of 80 patients presenting with arytenoid granuloma. The demographic and clinical characteristics were also analyzed.

Results: Arytenoid granulomas exhibited a male predominance with male: female ratio being 3.7:1. A total of 73 patients (91.25\%) had unilateral granuloma, whereas seven patients (8.75\%) had bilateral granuloma. Of the unilateral granulomas, $46(57.5 \%)$ patients presented with left-sided granuloma, and $27(33.8 \%)$ presented with right-side granuloma. The subtype of process granuloma (PG) was seen in 43 patients (53.75\%) and BG in 37 patients (46.25\%). PG is the commonest type of granuloma in males, and BG is the commonest type of granuloma in females. The association of process granuloma with inter arytenoid pachydermia was statistically significant but not BG. All cases of vocal cord paralysis in our series had BG. The majority of vocal cord paralysis (42.8\%) cases with arytenoid granuloma showed severe overriding of arytenoid cartilage on the normal side.

Conclusion: Identifying the two subgroups of arytenoid granuloma, namely process and BG, is imperative as they guide the proper management of arytenoid granuloma.

Keywords: Arytenoid granuloma, Interarytenoid pachydermia, Videolaryngoscopy, Vocal process.

International Journal of Phonosurgery \& Laryngology (2021): 10.5005/jp-journals-10023-1224
\end{abstract}

\section{INTRODUCTION}

Arytenoid granulomas are benign proliferative lesions located in the posterior glottis. These lesions are most commonly centered over the tip of the vocal process. Chevalier Jackson is credited with the first description of arytenoid granuloma in 1928, which he termed "contact ulcer of the larynx." These lesions are synonymously described in different literatures as laryngeal contact ulcer, contact granuloma, vocal fold granuloma and postintubation granuloma, and vocal process granuloma., ${ }^{1,2}$ The arytenoid granulomas can appear as reddish or whitish lesions that may be sessile or pedunculated or appear ulcerative on the surface. They are commonly unilateral or sometimes bilateral, and biopsy usually reveals squamous hyperplasia with a proliferation of capillaries, fibroblasts, collagen fibers, and leukocytes. ${ }^{3,4}$ These changes characterize granuloma as inflammatory angiomatous lesion called pyogenic granulomas.

The optimal management of this condition is still a matter of debate. Treatment of this condition is directed against the underlying cause, but the cause remains obscure in many cases. Intubation, phonotrauma/hyperfunctional, or laryngopharyngeal reflux remains the primary cause of this condition. Sometimes there may be significant overlap between these primary causes or may be multifactorial or emerge as recalcitrant idiopathic granulomas in some. ${ }^{2}$

Symptomatic patients are initially managed with a conservative combination of empiric medical therapy to tackle laryngopharyngeal reflux and voice therapy to mitigate hyperfunctional vocal behaviors. ${ }^{5}$

Surgery (Cold steel or laser ablation) is usually done when these conservative measures fail. Other indications for surgery are to take a biopsy to rule out malignancy and in cases of airway

\footnotetext{
1,2Department of Laryngology, Dr Jayakumar's Institute of Laryngology, Thiruvananthapuram, Kerala, India

${ }^{3}$ Department of Laryngology, Madhavam Laryngology Clinic, Kochi,
} Kerala, India

Corresponding Author: Vivek S, Department of Laryngology, Dr Jayakumar's Institute of Laryngology, Thiruvananthapuram, Kerala, India, Phone: +91 9447776906, e-mail: viveksoman3@gmail.com

How to cite this article: Vivek S, Menon JR, Nair RM. Decision-making in Arytenoid Granuloma Management: An Insight into the Presentations and Etiology. Int J Phonosurg Laryngol 2021;11(2):61-65.

Source of support: Nil

Conflict of interest: None

obstruction posed by granuloma. Antibiotics, corticosteroids, post excision irradiation, and botulinum toxin $\mathrm{A}$ injection are the other treatment options used in different centers. Caroll et al. suggests augmentation of true vocal cord as an option or adjunct to treat vocal process granuloma (PG) due to hyperfunctional behaviors secondary to an incompetent glottis. ${ }^{6}$

In this study, we aim to classify arytenoid granulomas into two subclasses- one which arises from the vocal process described henceforth as process granuloma (PG) and the other seen posterosuperiorly on the body of arytenoid as body granuloma (BG). This helps to analyze and study the etiological factors of each of these subtypes. We hypothesize that the mechanisms leading to the formation of both these granulomas are different. We also believe that targeted treatment directed against each of these granulomas decreases the unpredictable results of the treatment and recurrences. 


\section{Materials and Methods}

The study was conducted following the ethical standards of the institutional research committee and with the 1964 Helsinki Declaration and its later amendments and comparable ethical standards. Informed consent of the participants was taken.

The retrospective analysis of videolaryngoscopy (VLS) recordings of the senior authors for 9 years were employed for the study. The laryngeal examination was performed using a Storz $90^{\circ}$ rigid endoscope (with stroboscope) with the patient comfortably seated and phonating a sustained vowel at habitual pitch and intensity. In cases of suspected muscle tension dysphonia flexible fiber-optic examination was done to complement the diagnosis. Two laryngologists reviewed the video recordings for confirmation of findings and diagnosis. A total of 80 patients were recruited for this study. Patients with complaints of hoarseness, an abnormal sensation in the throat, or excess throat mucus and diagnosed as having arytenoid granuloma by using VLS and also cases of intubation granulomas were included in this study. Diseases of the immune system, deformities, and tumors of pharynx and larynx, and patients not consenting for video laryngoscopy examination were excluded from the study.

\section{Statistical Analysis}

The analysis data was entered into a Microsoft Excel spreadsheet and then analyzed by SPSS 26 version (veni 27.0. SPSS I, Chicago, IL, USA). Data were summarized, and frequency and percentage were calculated for categorical variables, and for testing the association, Chi-square test was used. The significance level was 0.05. $p$-value $\leq 0.05$ was considered as significant.

\section{Results}

Eighty patients with a diagnosis of arytenoid granuloma were included in the study. Of these, 63 patients (78.75\%) were males, and 17 patients (21.25\%) were females (Fig. 1). The male: female ratio in our study was 3.7:1. Unilateral granuloma was seen in 73 patients $(91.25 \%)$, whereas bilateral granuloma was seen in seven patients (8.75\%). Of the unilateral granulomas, 46 (57.5\%) patients presented with left-sided granuloma, and 27 (33.8\%) patients presented with right-side granuloma (Table 1). The subtype of PG was seen in 43 patients (53.75\%) and BG in 37 patients (46.25\%). The PG was seen in 39 males (61.9\% of males), and BG was seen

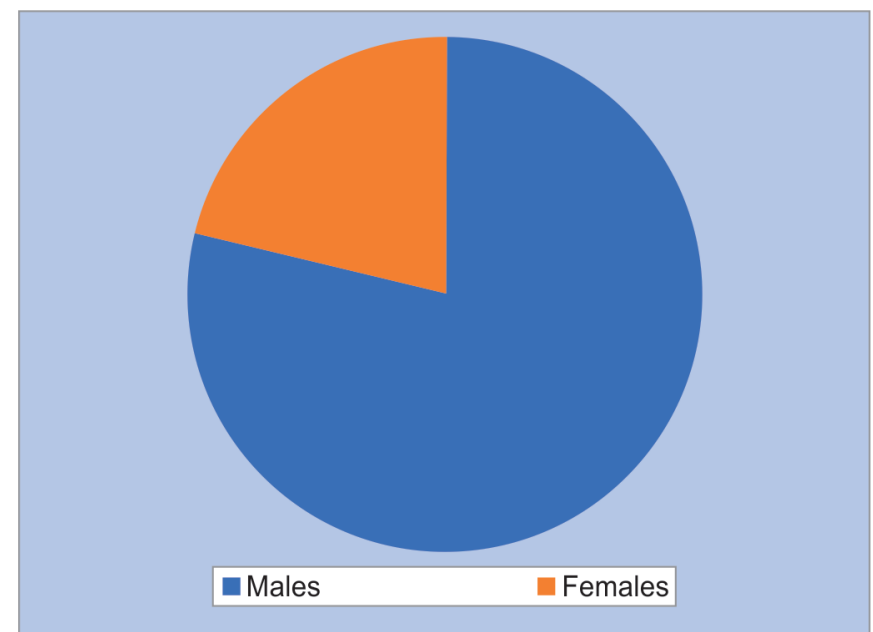

Fig. 1: Sex distribution of the study population in 13 females ( $76.5 \%$ of females) with $p$ value $<0.05$ (Table 2 ). So the PG is the commonest type of granuloma in males, and BG is the commonest type of granuloma in females. Phonatory gap was noted in 33 patients (41.3\%), and ventricular band adduction was identified in 47 patients (58.8\%). We have noted two types of movements of arytenoid cartilage in this study. One is overriding arytenoid (ORA), which is characterized by arytenoid adduction asymmetry of the two arytenoids identified by nonalignment of the glottic and interarytenoid axis. Another type of arytenoid movement is the over-adducting arytenoid (OAA), when both arytenoids simultaneously contract so that both vocal processes are not viewed at the peak of adduction. ORA was seen in 43 patients (53.8\%) and OAA was seen in 19 patients (23.8\%). We posit a grading system for ORA, which is shown below:

- Grade 1: Anterior crus of overriding arytenoid reaches up to the posterior end of cuneiform cartilage

- Grade 2: Anterior crus of overriding arytenoid reaches between posterior and anterior end of cuneiform cartilage

- Grade 3: Anterior crus of overriding arytenoid reaches beyond the anterior end of cuneiform cartilage.

The frequencies of each of the grades of arytenoid overriding in our study are shown in Table 3.

Of the seven patients who show bilateral granuloma, four patients (57.1\%) have OAA cartilage with $p$ value $=0.011$. So OAA may be considered as a causative factor for bilateral granuloma (Table 4).

Interarytenoid pachydermia is one of the laryngeal signs seen in laryngopharyngeal reflux disease. When PG and BG were analyzed separately, we found that PG was associated with inter arytenoid pachydermia with $p$ value $=0.004$. There was no such association in the case of BG (Table 5). Interestingly we found that all cases of vocal cord paralysis in our series had BG. Severe ORA was seen in three cases (42.85\%) of vocal cord paralysis, moderate ORA was seen in 1 case $(14.28 \%)$, mild ORA was seen in 1 case (14.28\%), and no overriding was seen in two cases (28.57\%). The side of granuloma was not associated with the side of ventricular band adduction (with $p$ value $=0.844$ ). Neither PG nor BG is associated with ORAs with $p$ values, respectively 0.879 and 0.411 . The prominence of hyoid bone during phonation of "ee" was noted in seven patients, out of which five patients showed prominent hyoid bone on the right side, and two patients showed prominent hyoid bone on the left side. Along with granuloma, vocal cord cyst was noted in two patients, and sulcus vocalis was noted in two patients in our study.

\section{Discussion}

The male:female ratio obtained in our study was 3.7:1 with a mean age of 44 . The youngest patient was 26 years of age, and the oldest patient was 71 years of age. In the study by Weing Chang et al., the male-to-female ratio was approximately $6: 1$, with the youngest subject being 9 years old and the oldest being 74 years old, with median age of 48.5 years. ${ }^{7}$ Yilmaz et al. included 20 patients with recurrent contact granuloma in their study and observed that they are nine times more common in men than females. ${ }^{8}$ The increased predisposition may be because males are more affected by age-related changes of the

Table 1: Side of granuloma

\begin{tabular}{lc}
\hline Right & 27 \\
Left & 46 \\
Both & 7 \\
\hline
\end{tabular}


Arytenoid Granuloma Presentations and Etiology

Table 2: Subtypes of arytenoid granuloma gender wise

\begin{tabular}{|c|c|c|c|c|c|}
\hline \multirow[b]{2}{*}{ Sex } & & \multicolumn{2}{|c|}{ Granulation tissue } & \multirow[b]{2}{*}{ Total } & \multirow[b]{2}{*}{$p$ value } \\
\hline & & Vocal process & Body of arytenoid & & \\
\hline \multirow[t]{2}{*}{ Male } & Count & 39 & 24 & 63 & 0.004 \\
\hline & $\%$ & 61.9 & 38.1 & 100.0 & \\
\hline \multirow[t]{2}{*}{ Female } & Count & 4 & 13 & 17 & \\
\hline & $\%$ & 23.5 & 76.5 & 100.0 & \\
\hline \multirow[t]{2}{*}{ Total } & Count & 43 & 37 & 80 & \\
\hline & $\%$ & 53.8 & 46.3 & 100.0 & \\
\hline
\end{tabular}

Table 3: Grades of overriding arytenoid cartilage

\begin{tabular}{lccc}
\hline & & Count & $\%$ \\
\hline Grade of overriding arytenoid cartilage & Nil & 37 & 46.3 \\
& Mild & 12 & 15.0 \\
& Moderate & 22 & 27.5 \\
& Severe & 9 & 11.3 \\
\hline
\end{tabular}

Table 4: Over-adducting arytenoid cartilage association with side of granuloma

\begin{tabular}{|c|c|c|c|c|c|c|c|}
\hline & & \multicolumn{3}{|c|}{ Side of granuloma } & \multirow[b]{2}{*}{ Total } & \multirow[b]{2}{*}{$x^{2}$} & \multirow[b]{2}{*}{ pvalue } \\
\hline & & Right & Left & Both & & & \\
\hline \multirow{2}{*}{$\begin{array}{l}\text { Presence of } \\
\text { over-adducting arytenoid } \\
\text { cartilage }\end{array}$} & Count & 2 & 13 & 4 & 19 & 9.113 & 0.011 \\
\hline & $\%$ & 10.5 & 68.4 & 21.1 & 23.8 & & \\
\hline \multirow{2}{*}{$\begin{array}{l}\text { Absence of over-adduct- } \\
\text { ing arytenoid cartilage }\end{array}$} & Count & 25 & 33 & 3 & 61 & & \\
\hline & $\%$ & 41.0 & 54.1 & 4.9 & 76.2 & & \\
\hline \multirow[t]{2}{*}{ Total } & Count & 27 & 46 & 7 & 80 & & \\
\hline & $\%$ & 33.8 & 57.5 & 8.8 & 100.0 & & \\
\hline
\end{tabular}

Table 5: Association of interaytenoid pachydermia with process granuloma and body granuloma

\begin{tabular}{|c|c|c|c|c|c|}
\hline \multicolumn{6}{|c|}{ Interarytenoid pachydermia with process granuloma } \\
\hline & Observed $N$ & Expected $N$ & Residual & $x^{2}$ & Pvalue \\
\hline Absent & 12 & 21.5 & -9.5 & 8.39 & 0.004 \\
\hline Present & 31 & 21.5 & 9.5 & & \\
\hline Total & 43 & & & & \\
\hline \multicolumn{6}{|c|}{ Interarytenoid pachydermia with body granuloma } \\
\hline & Observed $N$ & Expected $N$ & Residual & $x^{2}$ & Pvalue \\
\hline Absent & 15 & 18.5 & -3.5 & 1.32 & $0.25^{*}$ \\
\hline Present & 22 & 18.5 & 3.5 & & \\
\hline Total & 37 & & & & \\
\hline
\end{tabular}

larynx and may lead to more secondary hyperfunctional behaviors that further exacerbate an already traumatized vocal process. ${ }^{6}$ The presence of posterior chink at the vocal process level during phonation may protect against contact granuloma in women. However, contact ulcers resulting from endotracheal intubation are more common in females due to the thinner arytenoid perichondrium and narrow glottis, making them prone to injury. ${ }^{2}$ They also found that $75 \%$ of contact granuloma occurs at the medial face of the vocal process of the arytenoid and $25 \%$ in the body of the arytenoid at the posterosuperior aspect. $^{8}$ Our study found the incidence of PG (53.75\%) slightly more than BG (46.25\%). This paper is the first of its kind, which identifies the PG and BG separately to analyze the etiological basis of each.
Vocal granuloma usually follows injury to the posterior glottis. Hoffman et al. proposed causes of granuloma being mechanical and inflammatory. The mechanical cause may be either vocal trauma or nonvocal trauma like intubation injury and surgical trauma. Inflammatory type may be linked to GERD, postnasal discharge, infections, allergic, and idiopathic. Sometimes granuloma can arise due to the interaction of multiple causes. ${ }^{2}$ Caroll et al. found that $53 \%$ of vocal PG patients had an underlying glottal insufficiency (GI). They defined GI as a comorbid presence of true vocal fold atrophy, decreased true vocal fold mobility, or VF scar. Underlying glottic insufficiency should be promptly recognized, especially in the case of resistant vocal PG. ${ }^{6}$ 
Mechanism of injury is likened to the "hammer and anvil" where one vocal process hits hard against the other vocal process during the act of phonation, especially in loud phonation and hard glottal attacks. ${ }^{8,9}$ Voice rest is aimed at reducing this hammering action and hard glottal attacks. Out of the patients with bilateral granuloma, $57.14 \%$ were found to have OAA. It is logical to think that in OAAs, the violent collision results in bilateral granuloma.

The role of laryngopharyngeal reflux in laryngeal granuloma was suggested long ago. Compared with other laryngeal diseases, granuloma was detected more frequently in the LPR group. ${ }^{10}$ The study by Ogawa et al. revealed that reflux was an independent factor affecting the resolution of granuloma. ${ }^{11}$ In 1967, Cherry and Margulies used fluoroscopy to identify the association between vocal PG and laryngopharyngeal reflux. ${ }^{2}$ Later, Delahunty and Cherry, in an experiment, applied gastric juice daily for 20 minutes in the posterior glottic region to induce lesions consistent with vocal PG. ${ }^{13}$ However, Svensson et al. believed the association between reflux and contact granuloma was an indirect one because of the reactive hawking behavior arising due to chronic irritation induced by reflux laryngitis. ${ }^{14}$

We analyzed PG and BG separately for the association with reflux disease. Interarytenoid pachydermia was significantly associated with PG but not with BG in our study. This finding throws light into the different mechanisms in the causation of PG and BG. We believe that they should be treated as a separate entity when the management aspect of granulomas is considered.

Hillman et al. noted that abnormally high vocal cord closure velocity and collisions in patient with granuloma. According to their study mechanism for the development of granuloma is different from polyps or nodules. ${ }^{15}$

In our study, in cases where there is an underlying vocal cord paralysis, BG was seen in all cases. The study by Hoffman et al. also shared similar findings that granuloma occurred at a higher level on arytenoid rather than vocal process. This may be presumed due to the altered posterior glottic configuration directing contact forces to a high level. Apart from this vocal cord atrophy, paresis or scarring impart additional force to the vocal process on phonation and may result in granuloma. ${ }^{2}$

Intubation injuries usually affect the vocal process area. The reason for this is attributed to the thin mucoperichondrium, which lies above poorly vascularized cartilaginous vocal processes. ${ }^{16,17}$ The large size of the endotracheal tube, preexisting upper airway infection, and blind traumatic intubation are thought to be the cause of the development of granuloma after intubation. ${ }^{18}$

There are anecdotal reports suggesting allergy and postnasal discharge predisposing to granuloma. A logical explanation behind this would be a traumatic collision of vocal processes due to stimulus cough and hawking. ${ }^{2}$ McFerran et al. identified $26 \%$ of 34 cases in their series with vocal PG as idiopathic. ${ }^{19}$

The optimal treatment of this condition remains a challenge to the laryngologist. Treatment is mainly grouped into conservative and surgical management. Conservative measures include voice therapy and management of gastroesophageal reflux. Surgery (cold steel or laser ablation/photothermolysis) remains an option in recalcitrant cases when conservative measures fail, airway obstruction caused by granuloma, and in cases when the diagnosis is uncertain (i.e., ruling out malignancy). The extent of surgery varies from superficial excision to deeper resection with removal of the underlying cartilage Nasri et al., in 1995 was suggested for the first time the idea of using Botulinum toxin for the treatment of patients with contact granuloma. ${ }^{20}$ It can be injected into the region of the thyroarytenoid and lateral cricoarytenoid muscles to relax the adducting action on the arytenoid vocal process, thereby decreasing trauma of one vocal process to the other one. This injection relaxes the glottic sphincter. So in our series, this strategy may be used to treat resistant cases of BG as most of them occur due to the hammering action of arytenoids.

Vocal fold augmentation is an option in cases where there is an underlying GI. GI may predispose greater forces applied to the posterior glottis. ${ }^{15}$ These forces are redirected away from the vocal process when a successful vocal fold augmentation is performed.

Being a retrospective study by analyzing the VLS findings, no information could be collected regarding the chronicity of granuloma. The presence of LPRD was assessed only by inter arytenoid pachydermia. More precise measurements of laryngopharyngeal reflux disease (LPRD) like 24 hours $\mathrm{pH}$ monitoring would have accounted for a better selection of cases.

\section{Conclusion}

Arytenoid granulomas can manifest in two forms, namely PG and BG. In our study, PG contributed $53.75 \%$ and BG $46.25 \%$. Our study revealed that $P G$ is seen predominantly in males, and $B G$ is seen predominantly in females. Laryngopharyngeal reflux is a significant factor in the development of PG and not in BG. BG was noted in all cases where there is an underlying impaired vocal fold movement. We assume that these findings throw light into the management aspect of each type of granuloma. Antireflux therapy constitutes the mainstay of management of PG. Voice therapy in PG is aimed to correct overadduction of arytenoids vocal cords during closure and in BG by reducing supraglottic phenomenon whereas botulinum toxin injection can be employed in recalcitrant cases of BG. Vocal fold augmentation holds promise in the subset of population where there is an underlying $\mathrm{Gl}$ along with BG. However, more studies with a larger subset of study population are needed in this direction to prove these relationships conclusively.

\section{References}

1. Jackson C. Contact ulcer of the larynx. Ann Otol Rhinol Laryngol 1928;90:48-52. DOI: 10.1001/archotol.1935.00640030008001

2. Hoffman HT, Overholt E, Karnell M, et al. Vocal process granuloma. Head Neck 2001;23(12):1061-1074. DOI: 10.1002/hed.10014

3. Wenig BM, Heffner DK. Contact ulcers of the larynx. A reacquaintance with the pathology of an often underdiagnosed entity. Arch Pathol Lab Med 1990;114(8):825-828. PMID: 1695836.

4. Luzar B, Gale N, Klopcic U, et al. Laryngeal granuloma: characteristics of the covering epithelium. J Laryngol Otol 2000;114(4):264-267. DOI: $10.1258 / 0022215001905526$

5. Leonard R, Kendall K. Effects of voice therapy on vocal process granuloma: a phonoscopic approach. Am J Otolaryngol 2005;26(2):101-107. DOI: 10.1016/j.amjoto.2004.08.010

6. Carroll TL, Gartner-Schmidt J, Statham MM, et al. Vocal process granuloma and glottal insufficiency: an overlooked etiology? Laryngoscope 2010;120(1):114-120. DOI: 10.1002/lary.20656

7. Chang W, Xu W, Cheng L. Treatment of laryngeal contact granuloma: surgical therapy or conservative treatment. ORL 2019;81(56):348-353. DOI: 10.1159/000503975

8. Yılmaz T, Süslü N, Atay G, et al. Recurrent contact granuloma: experience with excision and botulinum toxin injection. JAMA Otolaryngol Head Neck Surg 2013;139(6):579-583. DOI: 10.1001/jamaoto.2013.3186

9. Horiguchi S, Suzuki M, Takagi H, et al. Clinical course of laryngeal granuloma without surgical treatment. Diagn Ther Endosc 2001;7:129-133. DOI: 10.1155/DTE.7.129

10. Akyildiz S, Ogut F, Varis A, et al. Impact of laryngeal findings on acoustic parameters of patients with laryngopharyngeal 
reflux. ORL J Otorhinolaryngol Relat Spec 2012;74(4):215-219. DOI: 10.1159/000340046

11. Ogawa M, Hosokawa K, Iwahashi T, et al. The results of Kaplan-Meier and multivariate analyses of etiological factors related to the outcome of combined pharmacological therapy against laryngeal granuloma. Acta Otolaryngol 2016;136(11):1141-1146. DOI: 10.1080/00016489.2016.1193891

12. Cherry J, Margulies SI. Contact ulcer of the larynx. Laryngoscope 1968;78(11):1937-1940. DOI: 10.1288/00005537-196811000-00007

13. Delahunty JE, Cherry J. Experimentally produced vocal cord granulomas. Laryngoscope 1968;78(11):1941-1947. DOI: 10.1288/00005537-196811000-00008

14. Svensson G, Schalén L, Fex S. Pathogenesis of idiopathic contact granuloma of the larynx. Results of a prospective clinical study. Acta Otolaryngol Suppl 1988;449:123-125. DOI: $10.3109 / 00016488809106393$
15. Hillman RE, Holmberg EB, Perkell JS, et al. Objective assessment of vocal hyperfunction: an experimental framework and initial results. J Speech Hear Res 1989;32(2):373-392. DOI: 10.1044/jshr.3202.373

16. Jackson C. Contact ulcer granuloma and other laryngeal complications of endotracheal anesthesia. Anesthesiology 1953;14(5):425-436. DOI: 10.1097/00000542-195309000-00001

17. Bishop MJ, Weymuller EA, Fink BR. Laryngeal effects of prolonged intubation. Anesth Analg 1984;63(3):335-342.

18. Ioannovich D. Bilateral polypoid granuloma of the larynx following endotracheal anesthesia: report of a case. AMA Arch Otolaryngol 1953:58(1):31-37. DOI: 10.1001/archotol.1953.00710040048004

19. McFerran DJ, Abdullah V, Gallimore AP, et al. Vocal process granulomata. J Laryngol Otol 1994;108(3):216-220. DOI: 10.1017/S0022215100126337

20. Nasri S, Sercarz JA, McAlpin T, et al. Treatment of vocal fold granuloma using botulinum toxin type A. Laryngoscope 1995;105(6):585-588. DOI: 10.1288/00005537-199506000-00005 